\title{
DIE OBVSG, VISUAL LIBRARY UND EINE STETIG WACHSENDE ANZAHL VON PUBLIKATIONEN UND NUTZERINNEN
}

\author{
von Silvio Wiese
}

Zusammenfassung: Digitale Repositorien sind im bibliothekarischen und wissenschaftlichen Bereich allgegenwärtig und entwickeln sich aufgrund neuer Anforderungen ständig weiter. Die Österreichische Bibliothekenverbund und Service GmbH (OBVSG) als Betreiberin der Repositoriumssoftware Visual Library hat umfassende Erfahrung mit der Koordination dieser Anforderungen: Von den Wünschen der NutzerInnen bis zur Umsetzung durch die verantwortlichen Firmen müssen vielfältige Interessen aufeinander abgestimmt werden. Der Beitrag beschreibt die Rolle der OBVSG als Betreiberin von Visual Library und aktuelle Vorhaben zur Weiterentwicklung der Software.

Schlüsselwörter: Konsortium; Langzeitarchivierung; Open Access; Repositorien

\section{THE AUSTRIAN LIBRARY NETWORK AND SERVICE LTD, VISUAL LIBRARY AND A STEADILY GROWING NUMBER OF PUBLICATIONS AND USERS}

Abstract: Digital repositories are central to both librarian and scientific work and keep evolving due to new requirements. The Austrian Library Network and Services Ltd. (OBVSG) is an application service provider of the repository software Visual Library. This includes the necessary coordination of both user requests and implementation by the companies responsible for the software. This paper describes the role of OBVSG as an application service provider of Visual Library and current plans for the further development of the software.

Keywords: Consortium; Digital Preservation; Open Access; Repositories

DOI: http://doi.org./10.31263/voebm.v71i1.1978

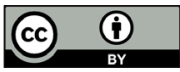

Dieses Werk ist lizenziert unter einer

Creative-Commons-Lizenz Namensnennung 4.0 International 


\section{Inhalt}
1. Einleitung
2. Publikationsmodul
3. Retrodigitalisierungsmodul
4. Weiterentwicklung
5. Fazit

\section{Einleitung}

Die Österreichische Bibliothekenverbund und Service GmbH (OBVSG) ist seit 2013 Betreiberin der Software Visual Library (VL), eines Produkts der Firma Semantics Kommunikationsmanagement $\mathrm{GmbH}^{1}$. Mit dieser Software können digitale Medien erfasst, erschlossen und präsentiert werden.

Die Software setzt sich aus verschiedenen Modulen ${ }^{2}$ zusammen und erfüllt eine Vielzahl an Aufgaben im Prozess des Digitalisierens und Publizierens. In den Modulen vereint Visual Library Funktionen zur Strukturierung und Verwaltung der Metadaten mit Werkzeugen der Datenaufbereitung, wie z.B. die PDF-Erzeugung und die Erzeugung von OCR-prozessierten Dateien.

In Österreich wurde eine konsortiale Lösung umgesetzt. Die OBVSG betreibt einen gemeinsamen Server für alle VL-Anwenderlnnen und die Workflows wurden österreichweit vereinheitlicht. Die Daten werden in den Verbundkatalog eingebracht und mit diesem synchronisiert. Dabei ist es möglich, den Volltextzugriff auf bestimmte Benutzerlnnenkreise einzuschränken, wobei einzelne Bereiche, wie etwa Bachelorarbeiten, von der Kommunikation mit dem Verbundkatalog ausgenommen werden können. In Österreich kommen die Module Retrodigitalisierung und Publikationsserver zum Einsatz. Der First Level Support für das Publikationsservermodul liegt direkt bei der OBVSG. Für das Modul der Retrokatalogisierung gehen Anfragen an die Firma Semantics, welche gegebenenfalls Fragen zu Metadaten und der Anbindung an den Verbundkatalog an die OBVSG weiterleitet.

Durch die Veränderungen im bibliothekarischen und wissenschaftlichen Bereich spielen digitale Repositorien eine größere Rolle als je zuvor. Die Repositorien unterscheiden sich stark, je nachdem, welche Nutzerlnnengruppen bedient und welche Medientypen gespeichert werden. Dabei sind die Anforderungen an Repositorien einem stetigen Wandel unterworfen, KundInnenwünsche und Nutzungsgewohnheiten ändern sich fortlaufend. 
Das erfordert von den BetreiberInnen von Repositorien ein Weiterentwickeln ihres Angebotes. Dabei müssen die vorhandenen finanziellen sowie zeitlichen Ressourcen so effizient wie möglich eingesetzt werden.

Im Augenblick wird Visual Library erfolgreich von 13 Einrichtungen in Österreich und einer ganzen Reihe von Institutionen in Deutschland und in der Schweiz genutzt. ${ }^{3}$ Allein in Österreich werden im Augenblick ca. 1,5 Millionen Digitalisate im Repositorium von Visual Library verwaltet. Etwa 130.000 Dokumente wurden bisher strukturiert ${ }^{4}$ und jeden Tag kommen neue dazu.

Diese große Anwenderlnnengemeinschaft in Österreich, Deutschland und der Schweiz bedeutet eine Vielfalt an KundInnenwünschen und garantiert damit die laufende Weiterentwicklung des Produkts.

\section{Publikationsmodul}

Mit dem Publikationsserver können Hochschulschriften, Open Access-Publikationen oder Präsentationen von Open Access-Journals und anderen Objekten automatisiert in einem zentralen Repositorium abgelegt werden. Die OBVSG versucht zusammen mit der Firma Semantics, neue Anforderungen aus dem KundInnenkreis nach wirtschaftlichen Kriterien und vorhandenen zeitlichen Ressourcen umzusetzen.

Die OBVSG bietet verschiedene Workflows, die bereits automatisiert ablaufen und ihren KundInnen das Arbeiten erleichtern.

1. Wissenschafterlnnen und Studierende können über ein Webformular ihre wissenschaftlichen Arbeiten in digitaler Form einbringen. Bibliotheksmitarbeiterlnnen unterziehen das Dokument einer technischen und formalen Qualitätsprüfung und geben dieses für die Veröffentlichung im Portal frei.

2. Aus einem bestehenden Campussystem kann via Schnittstelle ein Template ${ }^{5}$ in den Verbundkatalog einbracht werden. Nach der Qualitätsprüfung und Freigabe wird der Datensatz in den Publikationsserver importiert.

3. Besteht bereits eine Katalogaufnahme, werden durch das Hochladen des elektronischen Dokuments im VL-Manager automatisch die Metadaten aus dem Verbundkatalog importiert und das Dokument kann sofort freigegeben werden.

4. Open Access-Zeitschriften mit ihren besonderen Anforderungen bei der Erfassung und Verwaltung können in Visual Library in typischen Strukturen dargestellt und in individuellen PDFs bereitgestellt wer- 
den. Einzelne Aufsätze können in den Verbundkatalog exportiert und so auch dort wiedergefunden werden.

Allen diesen Workflows ist gemeinsam, dass diese Daten in verschiedenen von der OBVSG angebotenen Services automatisiert weiterverarbeitet werden. Dabei gibt es optionale und obligatorische Elemente.

Bei Freigabe des Dokuments in Visual Library wird unterschieden, ob dieses Dokument in den Österreichischen Verbundkatalog übernommen werden soll oder nur im lokalen Portal der jeweiligen Institution angezeigt wird. Soll der Titel in die Österreichische Verbundsuchmaschine ${ }^{6}$ eingebracht werden, kann optional eine Registrierung der URN ${ }^{7}$ vorgenommen werden. Seit 2014 betreibt die OBVSG einen URN-Service, der allen interessierten Institutionen und Personen auch außerhalb der VL-Community zur Verfügung steht. Damit können Dokumente einen eindeutigen und beständigen Identifier aus dem Namensraum „urn:nbn:at“ bekommen. Das ist ein wichtiger Beitrag zur Erhaltung der Langzeitverfügbarkeit.

Obligatorisch für die in die Verbundsuchmaschine eingebrachten elektronischen Publikationen ist die Verarbeitung durch den eDoc-Service ${ }^{8}$. Dabei werden elektronische Objekte wie Inhaltsverzeichnisse, Abstracts, Rezensionen, Klappentexte, Volltextdokumente und Bilder so aufbereitet, dass diese in der Verbundsuchmaschine suchbar werden.

\section{Retrodigitalisierungsmodul}

Ein weiteres Modul von Visual Library ist das Retrodigitalisierungsmodul ${ }^{9}$. Dieses wird stark genutzt und vereint eine Vielzahl an Softwarekomponenten, die den gesamten Workflow der Digitalisierung vom Anlegen des Metadatensatzes über das Kontrollieren der Bilddaten bis hin zur Bereitstellung in den jeweiligen Portalen abbildet. Das Ablegen der Objekte im Repositorium ist nur der kleinste Teil des Workflows.

Eine der Hauptaufgaben als Betreiberin von Visual Library ist die Organisation der Prozesse, die die Daten aus dem Repositorium in den verschiedenen Systemen sicht- und suchbar macht. Das Ziel ist eine höchstmögliche Automatisierung, um die Effizienz sowie die Fehlerfreiheit der einzelnen Workflows zu optimieren. Defizite und Grenzen der Software, die bei diesen Maßnahmen zutage treten, werden von der OBVSG dokumentiert und zur Behebung an die EntwicklerInnen von Visual Library weitergeleitet. Je nach Priorität werden diese sofort behoben oder in einem der folgenden Softwareupdates integriert. 


\section{Weiterentwicklung}

Ein zentrales Anliegen der VL-AnwenderInnen ist es, dass ihre Objekte adäquat dargestellt werden und gezielt nach ihnen gesucht werden kann. In den letzten Jahren ist der Wunsch nach Schnittstellen sehr stark gewachsen, die es erlauben, die Metadaten auch in andere Systeme zu übernehmen. Jedes Repositorium muss sich daran messen lassen, inwieweit es in der Lage ist, diese Anforderungen zu erfüllen. Leider ist es nicht möglich, die Anbindung an jedes gewünschte System zu verwirklichen, da auch wirtschaftliche Aspekte berücksichtigt werden müssen. Auf einem einmal jährlich stattfındenden Anwenderlnnentreffen der österreichischen Nutzerlnnengemeinschaft und einem Treffen aller deutschsprachigen AnwenderInnen wird zusammen mit den Entwicklerlnnen der Firma versucht, zukünftige Trends und Bedürfnisse aufzuzeigen und anschließend zu priorisieren. Danach wird gemeinschaftlich entschieden, in welche Aufgaben die nur begrenzt zur Verfügung stehenden finanziellen und personellen Ressourcen investiert werden. Außerdem besteht die Möglichkeit, sich für die eigenen Wünsche Verbündete bei den anderen AnwenderInnen zu suchen, um deren Umsetzung zu beschleunigen.

Durch die große Anzahl von AnwenderInnen und dieses demokratische Element ist sichergestellt, dass die wichtigsten Entwicklungen in der wissenschaftlichen Community implementiert werden und anschließend allen AnwenderInnen zur Verfügung stehen.

Für 2018 befinden sich folgende Hauptthemen auf der Entwicklungsliste von Semantics:

- Implementierung einer Schnittstelle zum Datenaustausch zwischen Bildrepositorien $\left(\mathrm{IIIF}^{10}\right)$. Damit kann VL auch an externe IIIF-Viewer angebunden werden.

- Umsetzung eines Moduls zur kooperativen Erschließung von Autographen

- Anbindung von externen XML-Editorensystemen (Oxygen), um projektspezifische Metadatenformate in VL über WebDAV einzubinden

- Schaffung der technischen Voraussetzungen für die Umsetzung von Responsive Design

- Implementierung einer aussagekräftigen Nutzungsstatistik

Für das von der OBVSG betreute Repositorium Visual Library wurde im Januar 2018 ein Workflow in Betrieb genommen, der archivierfähige ZipDateien inklusive aller notwendigen Metadaten erstellt. Dieser Workflow läuft jede Nacht und erfordert nur dann ein Eingreifen, wenn Fehler auftreten. Damit ist ein wichtiger Schritt getan, die Daten in den Repositorien 
auch für die Zukunft zugänglich zu halten. Diese Dateien eignen sich sehr gut für ein Langzeitarchivierungssystem.

Ziel dieses Prozesses ist es, die Originaldaten vor allem aus der Retrodigitalisierung für die Langzeitarchivierung vorzubereiten und anschließend aus dem Produktionssystem zu entfernen. Im System verbleiben die Derivate der Bilder in verschiedenen Auflösungen, die benötigt werden um die Digitalisate im Portal anzuzeigen. Das entlastet zum einen die Systeme und spart zum anderen Geld, da jede Kundin und jeder Kunde nur ein begrenztes Datenvolumen im Standardvertrag inkludiert hat. Die Zipkapsel enthält - neben Originaldateien und OCR-Daten - eine METS-Datei mit allen notwendigen Struktur- und Bildinformationen. Nachträgliche Änderungen an Bilddateien bzw. den Metadaten werden durch das Erstellen von inkrementellen „Deltakapseln“11 festgehalten. Bei einer Wiederherstellung der Stammdaten eines Katalogisats sind die zugehörige Masterkapsel und alle dazu erstellten Deltakapseln notwendig. Die Verbindung von Master- und Deltakapseln wird durch eine Datenbank verwaltet.

Aktuell arbeitet die OBVSG an der Verwirklichung eines lange gehegten Wunsches vieler Kundlnnen: Es soll ein Verfahren entwickelt werden, welches das Einbinden der DOI-Vergabe und deren Registrierung in die Workflows der VL-KundInnen ermöglicht. Ebenfalls prüft die OBVSG den Aufwand, die Metadaten so aufzubereiten, dass diese im Rahmen der bestehenden Workflows automatisch in Fremdsysteme, wie z.B. das DOAJ ${ }^{12}$, eingebracht werden können.

\section{Fazit}

Zusammenfassend kann gesagt werden, dass die OBVSG ihre ganze Bandbreite an Expertise über alle Abteilungen hinweg einsetzt, um den Ausbau und die Weiterentwicklung ihres Serviceangebotes rund um das von ihr betreute Repositorium voranzutreiben. Dazu gehören zum einen die konzeptionelle Mitarbeit bei der Weiterentwicklung von Visual Library, welche sehr stark von den verantwortlichen Firmen und ihrer Anwenderlnnengemeinschaft vorangetrieben wird, und zum anderen die Entwicklung von Diensten, die die Nutzbarkeit der vorhandenen Daten im Repositorium verbessern. Ein Schwerpunkt der OBVSG ist daneben das Entwickeln von Workflows, die das automatische Einbringen der Metadaten in weitere Fremdsysteme ermöglicht.

Für die Unterstützung möchte ich mich bei Gabriele Höfler und Mathis Kronschläger bedanken. 
Silvio Wiese, MA

Die Österreichische Bibliothekenverbund und Service GmbH (OBVSG) E-Mail: silvio.wiese@obvsg.at

1 https://www.semantics.de (16. April 2018).

2 Publikationsserver, Retrodigitalisierung, Semesterapparat und Bibliographie

3 https://www.obvsg.at/services/visual-library (16. April 2018) und https://www.semantics.de/visual_library/referenzen (16. April 2018).

4 Als PDF hochgeladene Publikationen werden im VL-Manager in der jeweils gewünschten Tiefe strukturiert, das heißt Vorwort, einzelne Aufsätze etc. werden mit Metadaten versehen.

5 Ein vordefiniertes Set an Feldern, wie Autor, Titel, Ort usw., welche mindestens ausgefüllt sein müssen.

6 http://search.obvsg.at/primo_library/libweb/action/search. do?vid=OBV (16. April 2018).

7 https://www.obvsg.at/services/urn-resolver (16. April 2018).

8 https://www.obvsg.at/services/edoc (16. April 2018).

9 https://www.semantics.de/visual_library/retrodigitalisierung (16. April 2018).

10 International Image Interoperability Framework (IIIF): http://iiif.io (16. April 2018).

11 Die neue Version wird also nicht vollständig nach Visual Library geladen. Die sogenannten „Deltakapseln“ beinhalten lediglich die Differenz zwischen der bereits vorhandenen und der neuen Version, wodurch erhebliche Mengen an Speicherplatz gespart werden können.

12 Directory of Open Access Journals (DOAJ): https://doaj.org (16. April 2018). 